\title{
An Efficient Multicast Routing Technique Using Genetic Algorithm over WDM Mesh Networks
}

\author{
Subhendu Barat, Soumitra Ghosh, Tanushree Dutta, and Tanmay De, Member, IACSIT
}

\begin{abstract}
Multicast Communication over an optical fiber network is treated as a major problem in optical domain. As the need of one-to-many communication is increasing day by day, researchers are trying to devise novel and efficient approaches to solve this problem. Although the most of the efforts done in this field is heuristic in nature, evolutionary approaches are also started to be used to solve this as an optimization problem. In this paper we have used Genetic Algorithm to find an efficient multicast route (tree) to establish a multicast connection in WDM mesh network. We have proposed a novel fitness function which can optimize multiple objectives: reaching maximum no. of destinations, minimizing usage of optical channels, minimizing usage of splitters, very efficiently. We have established the truth of our claim by simulating our proposed algorithm over various optical fiber networks.
\end{abstract}

Index Terms-Fitness function, genetic algorithm, multicast routing, light tree, splitter.

\section{INTRODUCTION}

Modern world is heavily dependent on information. Data exchange is becoming vital day by day, and hence faster and safer modes of data communication are becoming a key research issue. As the needs of industry and society are drifting towards multimedia information, data exchange is becoming bandwidth sensitive. With the advent of faster processors, data exchange speed is also needed to be faster, leading towards the requirement of high bandwidth faster mode of communication. Optical Fiber is thus becoming the backbone of modern day data communication. Application of Wavelength Division Multiplexing (WDM) [1] technique facilitated the transmission of multiple optical signal over same optical channel, thus a WDM optical fiber network can support more network traffic more efficiently.

Today, the majority of Internet applications rely on point-to-point transmission. The utilization of point-to-multipoint transmission has traditionally been limited to local area network applications. Over the past few years the Internet has seen a rise in the number of new applications that rely on multicast transmission. Multicast IP conserves bandwidth by forcing the network to do packet replication only when necessary, and offers an attractive alternative to unicast transmission for the delivery of network ticker tapes, live stock quotes, multiparty video-conferencing, and shared whiteboard applications (among others). It is

Manuscript received June 15, 2014; revised December 15, 2014.

Subhendu Barat, Soumitra Ghosh, and Tanushree Dutta are with the Department of Computer Science \& Engineering, NSHM Knowledge Campus Durgapur Group of Institutions, West Bengal, India (e-mail: barat.subhendu@gmail.com).

Tanmay De is with the Department of Computer Science \& Engineering, National Institute of Technology Durgapur, West Bengal, India. important to note that the applications for IP Multicast are not solely limited to the Internet. Multicast IP can also play an important role in large distributed commercial networks. The problem to establish a multicast connection over optical fiber network is termed as Multicast Routing and Wavelength Assignment (MRWA).

Multicast Routing and Wavelength Assignment problem can be segmented in two sub-problems: Multicast Routing (MR), and Wavelength Assignment (WA). Multicast Routing is a technique implemented in WDM optical networks, where dedicated paths are established between a source and a set of destinations. In this phase a route is found out over the network topology, which is basically a famous NP Hard problem in graph theory named, Minimum Steiner Tree (MST) problem. After minding the tree which spans all the member nodes in a multicast request, wavelength channel is assigned on the branches of the computed tree, such that optical signal can flow through the assigned channels. This phase of the problem is termed as Wavelength Assignment problem. The concept of light-path is vastly implemented in unicast routing and wavelength assignment problems (single source, single destination communication), where a light-path is treated as a logical connection between a source and a destination node in optical layer. In multicast communication the connection, after being assigned a particular wavelength, is termed as light-tree [2]. The key difference among multiple light-paths and a single light-tree is: to create a light-tree some of the internal nodes in the route need to be capable of splitting or replicating incoming light signal into multiple copies of outgoing light signal, each channeled through different branches leading to different member (destination) nodes. The nodes which are capable of splitting a light signal into multiple copies are termed as Multicast Capable (MC) nodes or Splitter node. As splitting reduces signal strength of the outgoing message, it requires amplification, hence splitting is treated as a costly operation which incurs more operational cost. Whereas, in absence of splitting number of wavelength channels requirement may increase, which leads to transmission blocking due to lack of wavelength channels in the WDM optical fiber network. Hence an efficient routing strategy is needed to be devised, which not only minimizes optical channel requirement but also reduces splitter usage, simultaneously. Thus Multicast Routing problem is basically a multi-objective optimization problem.

As the search space for MR problem increases rapidly with the increase in size of network topology and/or number of destinations in a single session request, standard heuristic algorithms become computationally expensive to provide a close approximation. Hence non-deterministic algorithms like Genetic Algorithm (GA) can be used to find an efficient solution in definite time interval. GA mimics the natural 
genetic behavior which explores the solution space more efficiently and rapidly and thus becoming a recent trend in research. This motivated us to propose a solution for MR problem using genetic algorithm. In this paper we have tried to optimize multiple objectives using evolutionary algorithm: GA. Here we have devised a novel fitness function, in which we have used weighted sum approach to optimize multiple objectives simultaneously. In the subsequent sections, we have shown a brief summary of the researches already done on this field, and then we have formulated Multicast Routing problem as a multi-objective optimization problem. Later we have described the working principal of Genetic Algorithm and presented our proposed algorithm with example. We have shown how the fitness function is improving through generations, and the impact of change in genetic parameters like mutation probability over the change in fitness function. Finally we have compared our proposed algorithm with heuristic algorithm and concluded the performance of the proposed algorithm with analysis.

\section{PREVIOUS WORKS}

The multicast routing problem with sparse light splitting was proposed and solved by X. Zhang et al. [3], where the network has both MC and MI nodes. Four routing algorithms were proposed to find a source based multicast light-forest. A sharing based multicast routing (SBMR) algorithm [4] is proposed in with the objective to minimize the number of wavelength and splitting requirement. Sahasrabuddhe and Mukherjee [2] formulated the multicast problem as an optimization problem with one of two possible objective functions: for a given traffic matrix, (i) minimize the network-wide average message hop distance, or (ii) minimize the total number of transceivers in the network. They solved the problem as a mixed-integer linear programming problem. There have been several studies on the optimization of split node placement in optical multicast networks. Ali [5] considers split node placement problems for static and dynamic traffic in wavelength-routed networks. Poo and Zhou [6] addressed the multicast wavelength assignment (MC-WA) problem in wavelength-routed WDM networks with full light splitting and wavelength conversion. It makes the multicast wavelength assignment more flexible, covering different switching schemes and different assignment strategies.

Recently researchers are focusing to solve MRWA problem using non-deterministic techniques like genetic algorithm. In [7] the multicast routing under delay constraint problem is considered in a WDM network with different light splitting. It firstly reduces the problem to the MST problem. Then it solves the problem by well-designed genetic algorithms. In [8] a multi-population parallel genetic algorithm is used for MRWA problem. The GA presented in the paper is hybridized with simulated annealing technique. A genetic routing algorithm [9] is proposed which exploits a combination of the alternate paths generated a priori for the given set of multicast requests. The main objective of this work is to minimize the number of split-capable nodes in the network for a given set of multicast requests. A resource constrained multicasting is proposed in [10] using Genetic Algorithm. The major contribution in the paper is that the authors have taken multiple objectives in account including QoS parameter like delay and network resource parameters like splitters, optical channels while generating light-tree for each multicast session request.

From the survey over the literature, it is very prominent that most of the solutions proposed in the domain of multicasting are heuristic in nature. In absence of MC nodes in all the nodes in the network, i.e. in sparse splitting environment, authors are shifting towards light-forest approach, which is a collection of sub-light-trees to establish a single optical multicast connection. The major disadvantage of light-forest over light-tree is, the former requires multiple transmitters to establish single multicast session, which is a costly physical parameter. The evolutionary approaches to solve multicasting problem in WDM network, is a new approach and need further research. Even many of the works, as discussed in this section, are not purely evolutionary. In many works, a hybrid approach is used, or only a part of the computation is only evolutionary. The most of the pure GA based approaches are mainly used in wavelength assignment phase, while routine phase is still dominated by heuristic approaches. In this paper we have proposed a pure simple genetic algorithm based algorithm to solve the multicast routing problem in WDM mesh network.

\section{PROBLEM FormULATION}

In this paper we are trying to solve multicast routing problem with an objective to maximize number of destinations reached and minimize number of wavelength channels to be used to establish a single multicast session. We also try to minimize splitting and splitters required in a WDM mesh network simultaneously. We can formalize this problem using the following mathematical formulation: We can assume a physical WDM mesh network is abstracted as a bi-directional graph $G(V, E)$, where $V$ is the set of vertices representing the nodes and $E$ is the set of edges representing the optical fiber links connecting different nodes in the physical WDM network. Our motto is to find a tree $t_{k}\left(V_{k}, E_{k}\right)$ for a multicast session request $r_{k} \in R$, where $R$ is the set of multicast requests for which dedicated optical sessions are to be established. The solution $t_{k}\left(V_{k}, E_{k}\right)$ is chosen such as all the destinations $d \in t_{k} \mid \forall d \in D_{k}$, where $r_{k}\left(s_{k}, D_{k}\right)$ is a multicast session request with source $s_{k}$ and member destination set is $D_{k}$.

\section{A. Assumptions}

In this work we have assumed some network properties which will hold in the underlying physical WDM network.

1) All the nodes (router/switch) in the network are multicast capable (MC), i.e. all the nodes have full splitting ability. An input signal entered in a node can branch out into multiple outgoing signals.

2) A source node can use a single transmitter to transmit a single multicast message. If branching is required at the source node, it will split the signal into multiple copies using splitters at the node.

3) No node in the network has wavelength conversion capability.

4) The traffic pattern is static, i.e. all the multicast session requests are known a priori. 
5) The network is free from node and/or link fault.

\section{B. Objective}

The motto of this work is to generate a set of multicast trees for a given set of static traffic. While generating multicast trees for a set of multicast requests, our objective is to:

1) Maximize number of destinations connected by the solution routing tree

2) Minimize wavelength channel requirement for a single multicast session requests

3) Minimize request blocking due to unavailability of wavelength channels, thus maximize throughput.

\section{Constraints}

The following constraints need to be maintained while constructing the multicast tree:

1) All the leaf nodes of the solution tree must be destination nodes

2) The solution tree needs to be rooted at the source node

3) All the destination nodes need to be distinct and different from the source node

4) All multicast session requests must contain exactly one source node and atleast one destination node

\section{PROPOSED APPROACH}

Multicast Routing in WDM mesh network is proved as NP Complete problem, as this problem can be reduced into well-known NP Hard problem Minimal Steiner Tree (MST) problem. In graph theory, the MST problem is defined as a problem to find a minimally connected tree which spans over a selected set of nodes. As in multicast routing a connection need to be established between single source with multiple destination nodes, the problem can easily be converted into a MST problem. As no polynomial time algorithm can be devised which will always provide the best solution of this problem, approximation is a feasible approach which can be taken. As the network size and/or member set of the session request increases, the search space becomes huge, and deterministic searching technique tends to fail. Using non-deterministic search strategy, the exploration of the search space is possible, but the convergence is very hard to expect until and unless some guided randomization is applied. Genetic Algorithm is a guided randomized search technique, which follows Markov's chain, if elitism is maintained, and thus guarantees convergence. Here we have proposed an algorithm which uses SGA with elitism and the proposed novel fitness function guides the searching efficiently towards convergence. Before going to the details of our proposed algorithm, here we will give a very brief description on the key features of SGA and the genetic operators.

\section{A. Simple Genetic Algorithm (SGA)}

Genetic algorithms (GA) are the main paradigm of evolutionary computing. GA is inspired by Darwin's theory about evolution - the "survival of the fittest". In nature, competition among individuals for scanty resources results in the fittest individuals dominating over the weaker ones. GA is the ways of solving problems by mimicking processes which nature uses i.e. Selection, Crossing over, Mutation and Accepting, to evolve a solution to a problem. It is an intelligent exploitation of random search used in optimization problems. Although GA is randomized, it exploits historical information to direct the search into the region of better performance within the search space.

\section{B. Outline of the Simple Genetic Algorithm}

1) Start: Generate random population of $n$ chromosomes, where each of the chromosomes is an encoded solution and $n$ such solutions form a generation.

2) Fitness: Evaluate the fitness $f(x)$ of each chromosome $x$ in the population, where $f($.$) is the fitness faction,$ which is defined as per the objective of the problem

3) New population: Create a new population by repeating following steps until the new population is complete.

- Selection: two parent chromosomes from a population according to their fitness (better the fitness, bigger the chance to be selected) are selected for mating

- Crossover: With a crossover probability, crossover the parents' genetic information to form new offspring (children). If no crossover was performed, offspring is the exact copy of parents.

- Mutation: With a mutation probability, mutate new offspring at each locus (selected position in chromosome), to explore new regions in the search space or to get rid of the stuck at local optima

- Accepting: Place fitter solutions in the new population.

4) Replace: Use new generated population for the next run of the algorithm replacing the older population.

5) Test: If the end condition is satisfied, stop, and return the best solution in current population.

6) Loop: Go to step 2

\section{Proposed Algorithm}

In our proposed approach we have used the following genetic construct and operators:

1) Encoding: We have used binary encoding scheme to represent a solution tree in the form of a binary chromosome. We have assumed the size of the chromosome is the number of edges in the network. If a particular edge $e i$ belongs to the solution tree $\left(E_{k}\right)$, the gene $g_{i}$ belongs to the chromosome is set to 1 , else its value is 0 .

$$
\begin{aligned}
& g_{i}=1 \text { if } e_{i} \in E_{k} \\
& g_{i}=0 \text { otherwise }
\end{aligned}
$$

2) Crossover: We have used single point crossover and the crossover point is selected at random. Two selected chromosomes will crossover with a predefined crossover probability. A single point crossover can be shown as in Fig. 1:

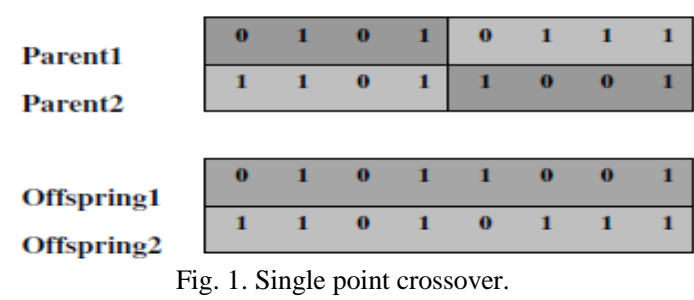

3) Mutation: A binary mutation is used with a predefined 
mutation probability. If a particular gene in the chromosome is mutated then it corresponding value will be flipped, i.e. 0 will be 1 and 1 will be 0 . The mutation is shown in Fig. 2.

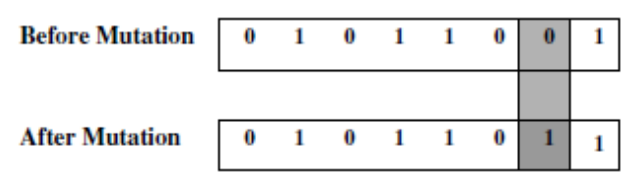

Fig. 2. Binary mutation.

4) Fitness Function: In any GA, fitness function is the most vital one. A fitness function quantizes how efficient a solution is. In our formulated problem, our objective is to reach most of the destinations which are part of the multicast session request. In most of the cases a transmission is said successful when all the intended recipients are able to receive the transmitted message. Hence this is a vital objective in multicasting. Similarly, as physical optical fibers are having finite number of wavelength channels, economic usage of optical fiber channels is required. A solution tree with lesser number of branches will require lesser number of wavelength channels to be assigned to establish a session. Hence we not only require more number of destinations (preferably all the destinations in the member set) to be connected through a single multicast tree but also, requires minimum number of branches in the solution tree.

These types of optimization problems are commonly termed as multi-objective optimization problem. In our proposed algorithm we have used proposed a weighted sum approach of multi-objective optimization. In weighted-sum approach, for each of the objective a weight will be assigned and these weights will signify how much effect of the solution's fitness corresponding to those objectives will be shown in the overall fitness of the solution.

Here, we have proposed a tunable SGA model, where the weight of a particular objective is tuned as per the user's requirement. Our formulated fitness function is as follows:

$$
\begin{aligned}
\text { Fitness }=(\alpha * & \left.d_{-} \text {count }\right) \\
& +\left(\beta /\left(x_{-} \text {count }-y_{-} \operatorname{count}+1\right)\right) \\
& +\left(\gamma /\left(y_{-} \text {count }+1\right)\right)
\end{aligned}
$$

where, $\alpha, \beta$, and $\gamma$ are weights, $d_{-}$count is the number of destinations reached by the solution tree, $x_{-}$count is the number of genes which are set to 1 , i.e. number of branches which are part of the solution, and $y_{-}$count is the number of branches which are reachable from the source node, i.e. the size of the connected tree rooted at the source node. The first clause in the equation tries to maximize number of destination reached. The second clause in the equation tries to minimize redundant edges in the solution, i.e. which are part of the solution but not the part of the tree rooted at the source node. The third clause in the equation minimizes the tree size, i.e. minimizes no. of wavelength channels required. Varying the $\alpha, \beta$, and $\gamma$ we can prioritize a particular objective over the other, and once the priority is set, after certain generations of the process, we will get efficient solutions which optimize our stated objectives.
5) Elitism: Elitism is an optional mechanism associated with SGA. In our approach we have used elitism, by retaining the best solution of a generation to pass through the next generation without any alternation. The elitism guarantees that the best fitness value of a generation will never go down in the successive generations. This property is very vital when we can formulate our fitness function in such a way that a growing fitness value necessarily leads to a better solution. In our proposed approach, we have formulated our fitness function in such a way, that given a priority to a particular objective over others (by assigning weights), a better fitness value always indicate a better solution and thus applying elitism we actually get a Markov's chain which signifies that our algorithm is going to converge towards global maxima. We should always remember though, multi-objective optimization can only provide a parreto optimal font, when we cannot prioritize any objective over other. Hence the convergence is true only when there exists a well-known set of weights.

The detailed pseudocode of our proposed algorithm is shown in Algorithm 1. Here we can see, if a multicast session request is supplied into the proposed Multicast Routing applying Genetic Algorithm (MRGA), the algorithm will return a multicast tree after a predefined number of generations. Here the number of generations is dependent on the size of population, nature of the solution, mutation and crossover ratio. The performance of the proposed MRGA is shown by simulation in the result section.

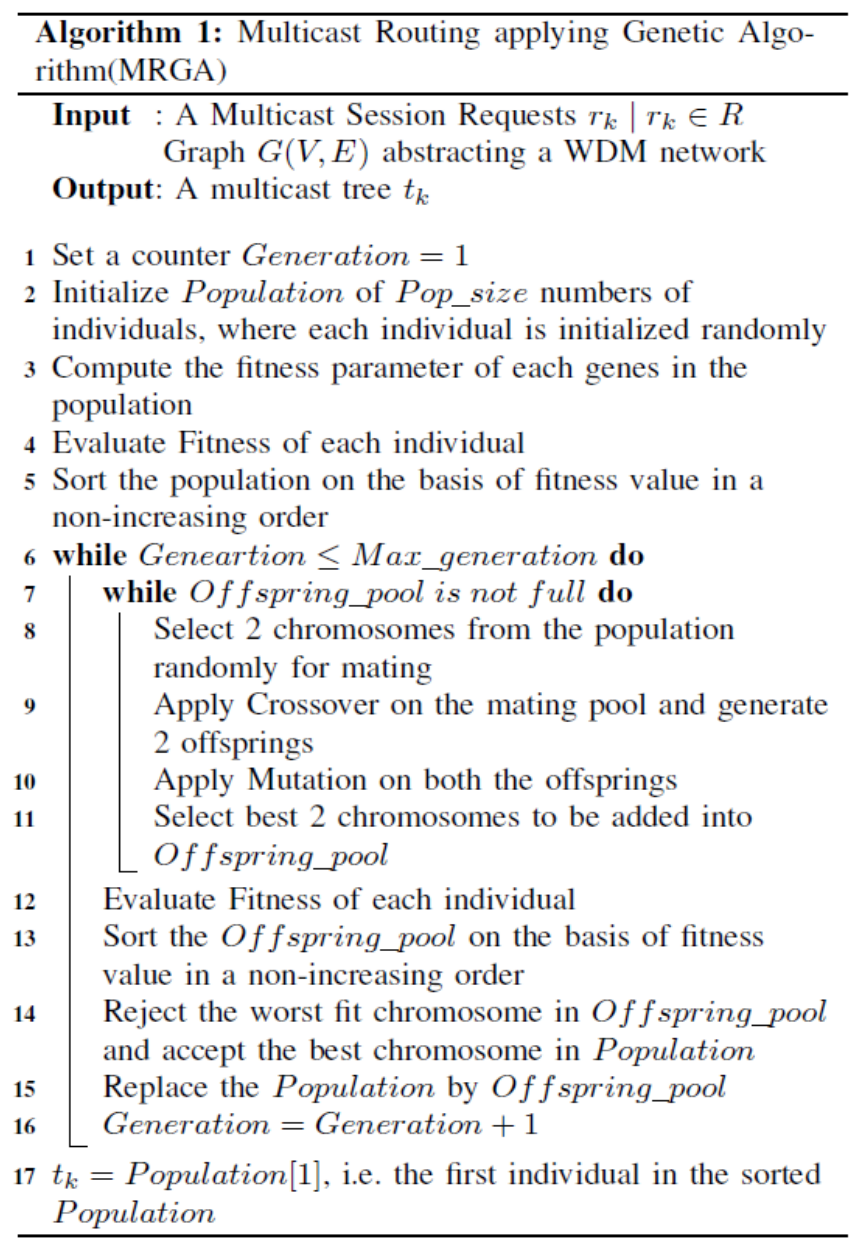




\section{EXPERIMENTAL RESULT}

To show the performance of our proposed algorithm, we have simulated the algorithm over 17-node Germany Network. We have taken random multicast session requests and checked the progression of fitness value through generations. We have also changed the network and genetic parameters to analyze the general behavior of our proposed algorithm and also to check the impact of the parameters over the performance of MRGA. We have also compared our proposed MRGA with a greedy algorithm (the most widely used heuristic).

\section{A. Simulation Model}

For the sake of simulation we have taken 17-node Germany network as the physical topology. 17-node Germany Network is a physical diagram of 17-districts in Germany interconnected to each other via links.

Networks are characterized by logical network topology and physical lengths of the cable ducts. The link lengths together with the number of nodes determine the network diameter in $\mathrm{km}$, which is defined as the longest shortest path with respect to length for any node pair. The German network has a diameter below $1000 \mathrm{~km}$

It consists of 17 nodes and 26 edges which can be represented as: $\left|V_{G E R}\right|=17,\left|E_{G E R}\right|=26$. As shown in Fig. 3, $V_{G E R}=\{0,1,2, \ldots, 16\}$ and $E_{G E R}=\{\langle 0\rangle,\langle 1\rangle$ $,<2>, \ldots,<25>\}$

We have assigned the above node numbers and edge numbers to identify distinctly each node and links in the actual network.

\section{B. Analysis of Results}

To check how the fitness value of the population in our proposed algorithm is varying with the generation, we have randomly selected some multicast session requests and plotted the best fitness values for each generation. From this result, as shown in Fig. 4, we can see fitness value increased in a consistent way as the generation progressed, and it tends to converge. In Fig. 4, we have taken 2 multicast session requests $\{12,2,5,6,9\}$ and $\{6,3,7,9,15\}$, where first node in the session request is the source node.

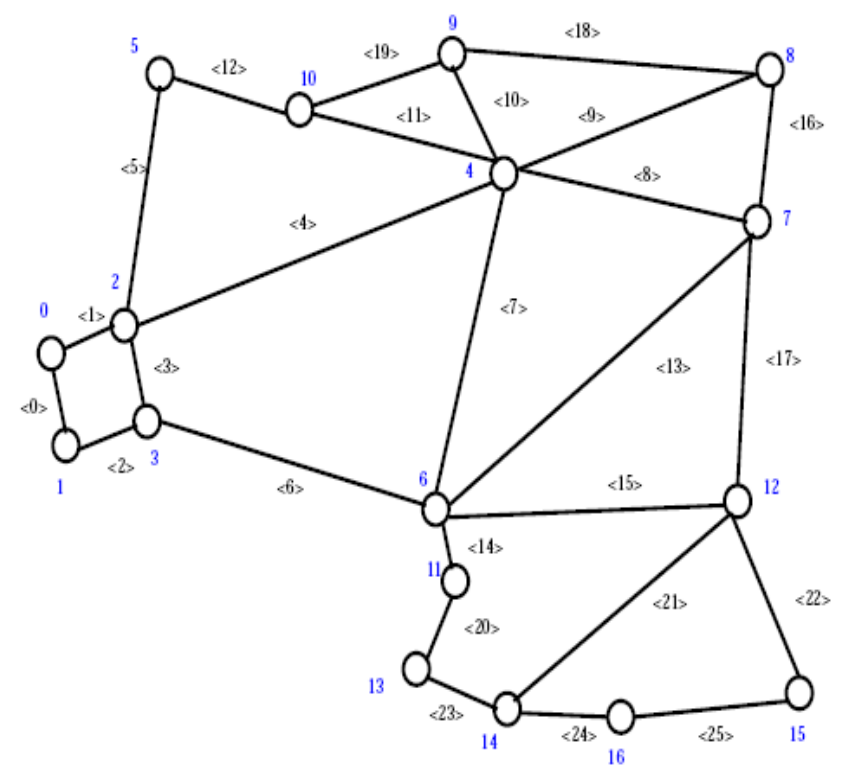

Fig. 3. 17-node Germany network.

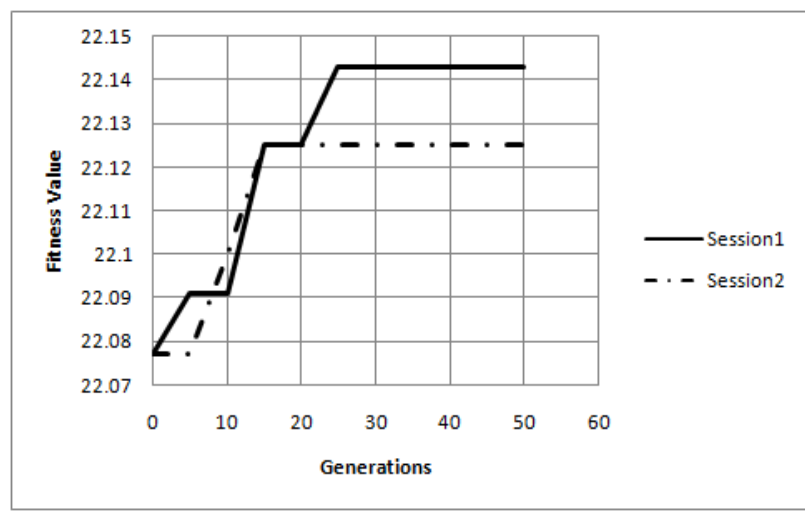

Fig. 4. Variation of fitness value over generations.

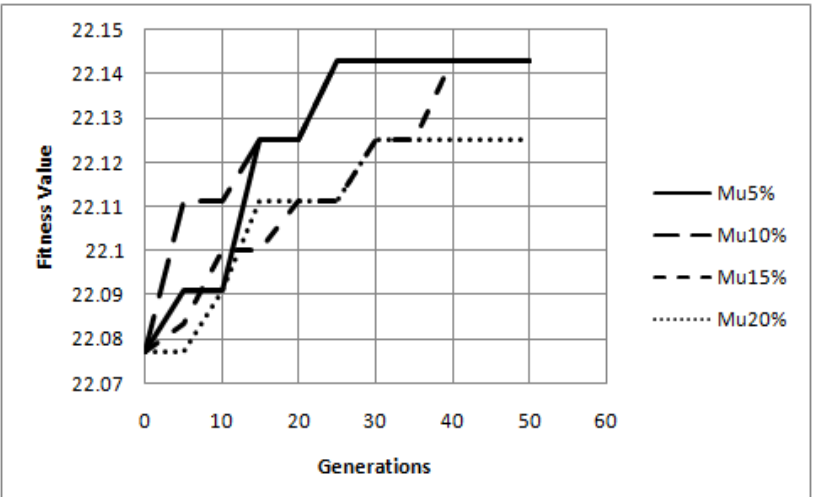

Fig. 5. Effect of mutation probability over convergence of fitness.

From Fig. 4 we can observe fitness values are increasing gradually with the generation, and never fall down due to elitism applied in our algorithm. It is also visible in both the random examples, fitness value tends to saturate after certain generations.

In Fig. 5 we have shown the relationship of mutation probability with the growth of fitness values per generation. In this figure, we observe as the mutation probability is increased from $5 \%$ to $20 \%$, the abruptness in the change in fitness value increases. The lower the mutation probability, the smoother the transitions in fitness values over generation, which requires longer execution time to reach the saturation, where as high mutation probability, may lead to greater disturbance in the genetic system and may overshoot the global optima.

We have taken 3 sets of random requests, each containing 10 multicast session requests, with number of member destinations are 4, 8, 12 respectively. Upon simulation with these request sets, we calculated the average values of tree size, i.e. wavelength channel requirements per session, number of destination reached per session and the amount of splitting requirement per session for each of the 3 sets. We have seen if we assume $\alpha$ is greater than summation of $\beta$ and $\gamma$, we always got all the destinations are reached. The reason behind it is, if we get a solution which has minimum redundancy, i.e. no redundant edge and minimum tree size, i.e. theoretically 0 , and practically 1 (if it contains only one destination and there exists a direct link between source and the sole destination), the contribution of the fitness due to these two clauses in the fitness function equation will be $(\beta+\gamma)$. Now if the solution does not connect all the destinations (assume $k$ destinations out of $n$ destinations are unreached), then its overall fitness value will be fitness $=$ 
$(\alpha *(n-k))+(\beta+\gamma)$. Now if, $\alpha>(\beta+\gamma)$, then fitness $<(\alpha *(n-k+1))$. Even if $k$ is minimum, i.e. 1 , fitness $<(\alpha * n)$. Whereas, any solution with all destinations reached will have minimum fitness, $(\alpha * n)$. Hence if there exists any solution which connected all the destinations, which is very likely in a randomized algorithm, that will override other solutions which is not connecting all the destinations. Hence we can guarantee the maximization of number of destination reached, by merely setting $\alpha>(\beta+\gamma)$. In our simulation also, we got the same result, as we set $\alpha=5, \beta=2, \gamma=1$.

The performance of our proposed GA is shown through Fig. 6 and Fig. 7, where we have shown how the Fitness values of each individual in a population is improving as the generation progresses. To demonstrate these Fitness Characteristics of the populations, we have taken a random population of size 50 which is evolved generation by generation until we reached saturation. For the simulation purpose we have taken a random multicast session request of random size, and plotted the individual fitness of each population for each generation.

In Fig. 6 we have shown the Fitness Characteristics for a multicast session request $\{5,\{2,3,7,8,11,12\}\}$ over Germany Network, where the source node is 5 and destinations are $\{2,3,7,8,11,12\}$. After $51^{\text {st }}$ generation, we can observe the entire pool is saturated, i.e. containing only one unique solution, or multiple unique solutions with same fitness value. From the figure, it is visible that initially the population was populated with varieties of solutions, among which many were not so good, as per fitness metric. As the generation advances, the successive populations contain fitter solutions, establishing the basic idea of GA: survival of the fittest. This fitness characteristic takes a "Funnel" like shape, which signifies an efficient convergence is achievable through our GA.

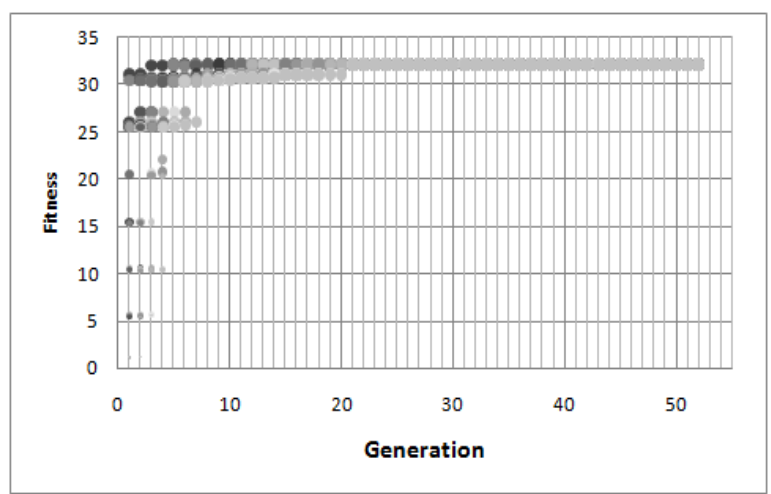

Fig. 6. Fitness Characteristic of population over generations.

In Fig. 7 we have plotted another Fitness Characteristic for the same above-mentioned multicast session request $\{5,\{2,3$, $7,8,11,12\}$ \} over Germany Network. Here we have performed a statistical analysis over the population as the generation proceeds. In this figure, we have plotted the maximum, minimum and the median of individual fitness values of the solutions in each population. The characteristic shows, the median curve and maximum curve coincide very rapidly, signifying greater number of solutions with higher fitness values. It also shows that minimum characteristic curve shows gradual improvement over generations, signifying the non-surveillance of week solutions in a population. Thus, the figure proves that our proposed fitness function is efficient enough to eliminate week solutions and enhances the exploration of better solutions over generations.

In Fig. 8 we have compared our proposed algorithm (MRGA) with heuristic (HUR) approach with respect to tree size, i.e. number of wavelength channels required. From the figure we can observe both the approaches require nearly same number of wavelength channels per session. We can minimize the tree size further, if we prioritize tree size, by assigning higher value of $\gamma$.

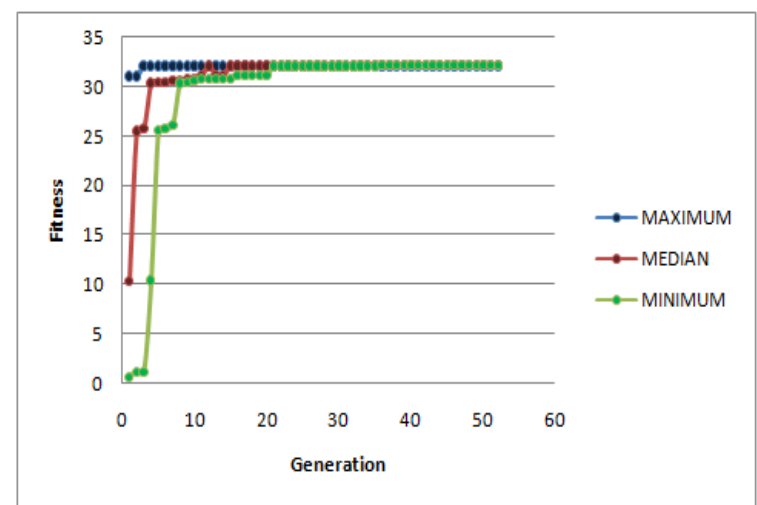

Fig. 7. Statistical Analysis of Fitness Characteristic of population.

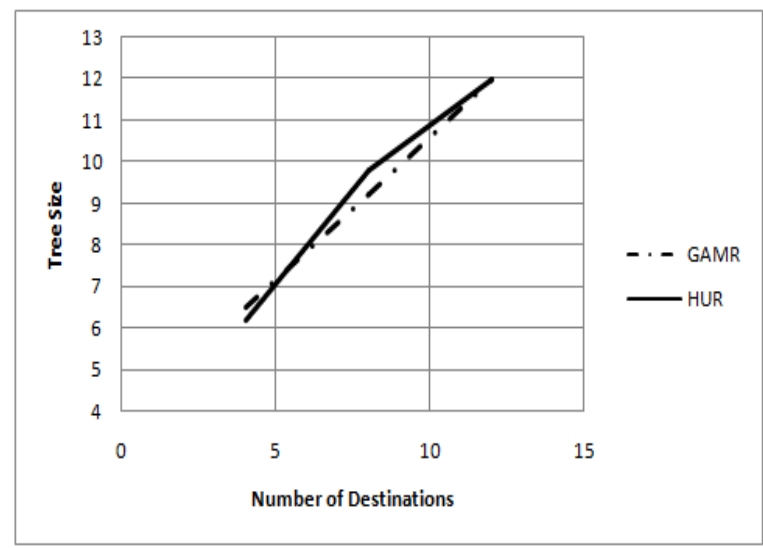

Fig. 8. Relationship of Tree size with number of destinations.

In Fig. 9 we have plotted splitting requirement with respect to number of destinations in the multicast session request. It is obvious from the result that, as we increase the number of destinations per session, amount of splitting also increases, but the increase is much less in case of MRGA than HUR, rather in all cases GA based routing requires lesser splitting. Thus our proposed algorithm is providing better splitting minimization than the heuristic algorithm.

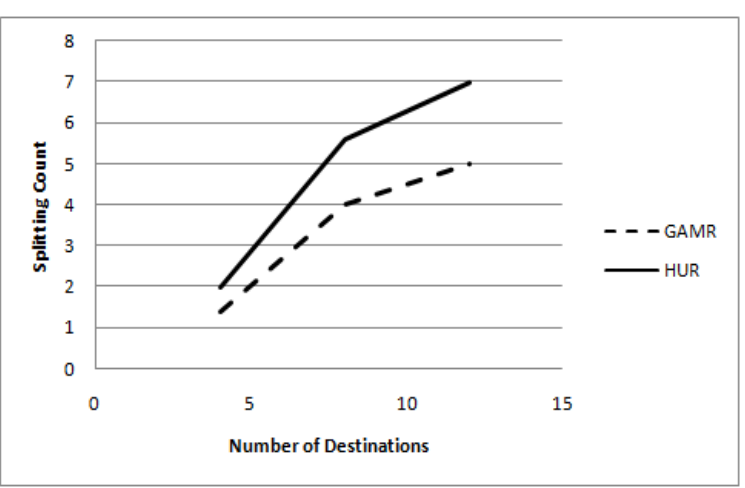

Fig. 9. Relationship of Splitting requirement per session with number of destinations. 


\section{CONCLUSION}

As optical fiber communication is playing a vital role as the backbone on modern communication, efficient routing and wavelength assignment algorithms are necessary so as proper resource management is possible. In this work, we have formulated multicast routing as a multi-objective optimization problem and proposed a Genetic Algorithm based MRGA to solve the MR problem. We have seen that our novel fitness function converges well with the generation, which indicates that our searching algorithm performs efficiently to reach the global optima. We have also shown the impact of genetic parameters like mutation probability on the convergence of fitness value over generations. The simulation results depict our proposed algorithm maximizes the number of destination reached very efficiently and also minimizes wavelength channel usage and also splitting requirement. Hence we can conclude that the proposed MRGA is an efficient Multicast Routing algorithm.

\section{REFERENCES}

[1] C. S. R. Murthy and M. Gurusamy, WDM Optical Networks: Concepts, Design, and Algorithms, Prentice-Hall India, 2002

[2] L. H. Sahasrabuddhe and B. Mukherjee, "Light-trees: Optical multicasting for improved performance in wavelength-routed networks," IEEE Communication Magazine, vol. 37, no. 2, pp. 67-73, Feb. 1999.

[3] X. Zhang, J. Wei, and C. Qiao, "Constrained multicast routing in WDM networks with sparse light splitting," in Proc. IEEE INFOCOM, 2000, vol. 3, pp. 1781-1790.

[4] S. Barat, A. K. Pradhan, and T. De, "A cost efficient multicast routing and wavelength assignment in WDM mesh network," in Proc. $10^{\text {th }}$ International Conference on Computer Information System and Industrial Management, 2011, vol. 245, pp. 65-73.

[5] M. Ali, "Optimization of splitting node placement in wavelength routed optical networks," IEEE Journal on Selected Areas in Communication, vol. 20, no. 8, pp. 1571-1579, Oct. 2002.

[6] G. S. Poo and Y. Zhou, "A new multicast wavelength assignment algorithm in wavelength-routed WDM networks," IEEE Journal on Selected Areas in Communications, vol. 24, no. 4, pp. 2-12, 2006.

[7] M. T. Chen and S. S. Tseng, "A genetic algorithm for multicast routing under delay constraint in WDM network with different light splitting," Journal of Information Science and Engineering, vol. 21, no. 1, pp. 85-108, 2005.

[8] H. Cheng, X. Wang, S. Yang, and M. Huang, "A multipopulation parallel genetic simulated annealing-based QoS routing and wavelength assignment integration algorithm for multicast in optical networks," Journal of Applied Soft Computing, vol. 9, no. 2, pp. 677-684, 2009

[9] J. H. Siregar, Y. Zhang, and H. Takagi, "Optimal multicast routing using genetic algorithm for WDM optical networks," Journal of IEICE Transaction in Communication, vol. E88-B, no. 1, 2005.
[10] S. Barat, A. K. Pradhan, and T. De, "A genetic algorithm for multicasting in resource constraint WDM mesh network," in Proc. 7th IEEE International Conference on Industrial and Information Systems (ICIIS), 2012, pp. 1-6.

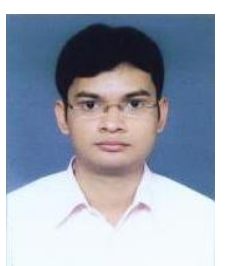

Subhendu Barat has completed master's degree of technology, in the year 2012, from the Department of Computer Science \& Engineering, National Institute of Technology (NIT) Durgapur, and bachelor degree of technology in the Department of Computer Science \& Engineering, from Asansol Engineering College under the West Bengal University of Technology, Kolkata, India, in 2010.

He is currently working as an assistant professor in the Department of Computer Science and Engineering at NSHM Knowledge Campus Group of Institutes. His research area is optical fiber communication.

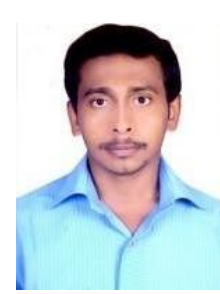

Soumitra Ghosh is a student of bachelor of technology in the Department of Computer Science \& Engineering, NSHM Knowledge Campus Durgapur Group of Institutions. His field of interest is optical fiber communication.

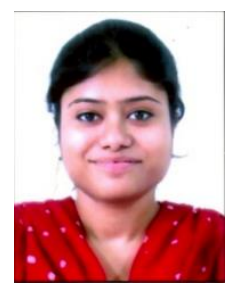

Tanushree Dutta is a student of bachelor of technology in the Department of Computer Science \& Engineering, NSHM Knowledge Campus Durgapur Group of Institutions. Her field of interest is optical fiber communication.

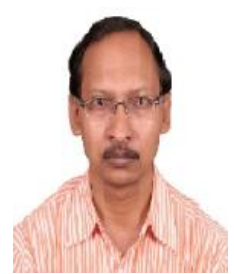

Tanmay De received the B.Tech. degree in computer science and engineering from University of Calcutta, India, in 1996, the M.Tech. degree in computer science and engineering from the Jadavpur University, India, in 1998 and the Ph.D. degree in Department of Computer Science and Engineering, Indian Institute of Technology (IIT) Kharagpur, India, in 2010.

Since 1998, he has been a faculty member of National Institute of Technology (NIT) Durgapur, India. His research interesdts include optical WDM networks, mobile ad hoc networks, and delay tolerant networks.

Dr. De is a member of IEEE and IACSIT. 